\title{
Role of Endogenous Atrial Natriuretic Factor in Acute Congestive Heart Failure
}

M. E. Lee, W. L. Miller, B. S. Edwards, and J. C. Burnett, Jr.

Cardiorenal Research Laboratory, Departments of Medicine and Physiology and Biophysics, Mayo Clinic and Foundation, Rochester, Minnesota 55905

\begin{abstract}
The current studies were designed to investigate the functional significance of elevated endogenous atrial natriuretic factor (ANF) in acute congestive heart failure (CHF). Integrated cardiorenal and endocrine function were measured in three models of acute low-output congestive heart failure with comparably reduced cardiac output (CO) and mean arterial pressure (MAP). Acute CHF was produced by rapid right ventricular pacing (group $\mathrm{I}, \boldsymbol{n}=5$ ) which decreases $\mathrm{CO}$ and increases atrial pressures and plasma ANF. In group II, $n=5$, thoracic inferior vena caval constriction (TIVCC) was produced to decrease venous return and $\mathrm{CO}$ but without increases in atrial pressure or plasma ANF. In group III, $n=5$, TIVCC was performed and exogenous ANF infused to achieve plasma concentrations observed in acute CHF. In acute CHF with increases in endogenous $A N F$, sodium excretion $\left(U_{\mathbf{N a}} V\right)$, renal blood flow (RBF), plasma renin activity (PRA), and plasma aldosterone (PA) were maintained despite decreases in $\mathrm{CO}$ and MAP. In contrast, TIVCC with similar reductions in $\mathrm{CO}$ and MAP but without increases in ANF resulted in decreases in $\mathrm{U}_{\mathrm{Na}} \mathrm{V}$ and $\mathrm{RBF}$ and increases in PRA and PA. Exogenous administration of ANF in TIVCC to mimic levels in acute CHF prevented sodium retention, renal vasoconstriction, and activation of renin and aldosterone. These studies demonstrate that endogenous ANF serves as an important physiologic volume regulator in acute CHF to maintain sodium excretion and possibly participate in the suppression of activation of the reninangiotensin-aldosterone system despite the stimulus of arterial hypotension.
\end{abstract}

\section{Introduction}

Atrial natriuretic factor (ANF) ${ }^{1}$ is a peptide hormone of cardiac origin which is released from the mammalian atria in response to atrial stretch $(1,2)$. Exogenous administration of

Address reprint requests to Dr. Burnett, Mayo Clinic, 200 First Street S.W., Rochester, MN 55905.

Received for publication 28 September 1988 and in revised form 21 June 1989.

1. Abbreviations used in this paper: ANF, atrial natriuretic factor; $\mathrm{CHF}$, congestive heart failure; $\mathrm{CO}$, cardiac output; $\mathrm{EP}$, experimental period; HR, heart rate; MAP, mean arterial pressure; PCWP, pulmonary capillary wedge pressure; PRA, plasma renin activity; RAAS, renin-angiotensin-aldosterone system; RAP, right atrial pressure; $R B F$, renal blood flow; $R P$, recovery period; $R V P$, rapid ventricular pacing; TIVCC, thoracic inferior vena cava construction.

J. Clin. Invest.

(C) The American Society for Clinical Investigation, Inc. 0021-9738/89/12/1962/05 \$2.00

Volume 84, December 1989, 1962-1966
ANF at physiologic as well as pharmacologic concentrations increases sodium excretion with inhibition of renin and aldosterone (3-5). In acute and chronic congestive heart failure (CHF), circulating ANF is increased reflecting enhanced cardiac release (6-13). Despite this elevation, no natriuretic response is observed. Thus, the functional significance of these compensatory responses in renal and endocrine function to increased circulating ANF is unclear.

In preliminary studies by Miller and co-workers (14), elevation of ANF in acute experimental CHF produced by rapid right ventricular pacing was unassociated with sodium retention or activation of the renin-angiotensin-aldosterone system (RAAS), despite the potent stimulus of decreased cardiac output and arterial pressure. Similarly, recent studies in rats with CHF produced by acute myocardial infarction with high circulating ANF associated with decreased cardiac output were unassociated with increases in renin and/or aldosterone $(6,7)$. These observations support the hypothesis that endogenous elevation of ANF in acute and chronic CHF may serve as a homeostatic mechanism to maintain renal excretory function and attenuate activation of the RAAS despite a reduction in arterial pressure.

To test this hypothesis, integrated cardiorenal and endocrine function were measured in two models of acute low cardiac output heart failure both with comparably reduced cardiac output and arterial pressure. Acute ventricular tachycardia produced by rapid right ventricular pacing represents a model associated with increased atrial pressure and plasma ANF. The second model employed was thoracic inferior vena caval constriction (TIVCC) characterized by normal atrial pressure and subsequent plasma ANF resulting from decreased venous return. A third group of dogs were studied with TIVCC in which exogenous ANF was infused to mimic plasma concentrations observed in group I with ventricular pacing and high endogenous ANF.

\section{Methods}

\section{Surgical procedure}

Experiments were performed on three groups of mongrel dogs of either sex weighing 14-26 kg. The dogs were fasted overnight before the acute experiment, but water was allowed ad lib. All dogs were maintained on a 150-meq/d sodium diet. Dogs were anesthetized with sodium pentobarbital (30 mg/kg) and maintained with supplemental doses as necessary.

After anesthesia, dogs were intubated and artificially ventilated (large-animal respirator, Harvard Apparatus Co., Inc., Millis, MA). Both femoral veins and the right femoral artery were catheterized with polyethylene tubing (i.d. 0.05 in., o.d. 0.09 in.). The right external jugular vein was isolated and a 7.5 French balloon-tipped thermodilution pulmonary artery catheter with right atrial port (model 93A-131F, American Edwards Laboratory, Santa Ana, CA) was advanced into the pulmonary artery.

A left flank incision was made and the left kidney, renal artery, and ureter were isolated. The ureter was cannulated with polyethylene 
tubing for collection of urine. An electromagnatic flow probe was placed on the renal artery and was connected to a flow meter (model FM 501D, Carolina Medical Electronics, Inc., King, NC). The remainder of the surgical procedures differed according to protocols described below.

Group 1. Rapid right ventricular pacing (RVP): the chest was opened at the left fifth intercostal space. An epicardial pacing wire was placed on the ventricular apex and then connected to a pulse generator (model 4563A, Medtronic Inc., Minneapolis, MN).

Group 2. TIVCC: after a right thoracotomy at the sixth intercostal space, an inflatable vascular occluder ( $20 \mathrm{~mm}$ i.d., IVM, Healdsburg, CA) was implanted around the thoracic inferior vena cava.

Group 3. TIVCC + ANF infusion: an inflatable balloon cuff was placed around the thoracic inferior vena cava through a right thoracotomy as described above.

\section{Protocols}

During the surgical preparation, an infusion of normal saline at 1 $\mathrm{ml} / \mathrm{min}$ was initiated and continued throughout the experiment via the right femoral vein catheter to replace the volume loss. After the surgical preparation, the animal was suspended in the physiologic position and allowed $1 \mathrm{~h}$ to equilibrate. During this equilibration period, infusion of inulin was begun via the left femoral vein catheter at $1 \mathrm{ml} / \mathrm{min}$. The amount of the inulin infused was calculated to achieve a plasma concentration of $50 \mathrm{mg} / \mathrm{dl}$. At the end of the equilibration period, a 15-min clearance (control) was collected. The remainder of the protocol differed among the three groups.

Group 1. Rapid RVP: after the control period, the right ventricle was paced at a rate to achieve a $15 \%$ reduction of the control mean arterial pressure (MAP). After 15 min of stabilization, a 30-min clearance (experimental period, EP) was performed. The rate of ventricular pacing was adjusted to control MAP at this level. At the end of the 30 -min clearance, ventricular tachycardia was terminated. $60 \mathrm{~min}$ after the termination of ventricular pacing, a 15-min clearance (recovery period, RP) was performed.

Group 2. TIVCC: after the control period, the thoracic inferior vena cava was constricted by inflation of the vascular occluder to achieve a $15 \%$ reduction of the control MAP and comparable decrease in cardiac output. After 15-min of stabilization, a 30-min clearance (EP) was collected. The volume of the vascular occluder had to be adjusted to maintain the MAP at the desired level. The vascular occluder was then deflated and a 15-min clearance (RP) was collected at $60 \mathrm{~min}$ after the deflation of the vascular occluder.

Group 3. TIVCC and ANF infusion: after the control period, the vascular occluder was inflated to achieve a $15 \%$ reduction in MAP; this occurred immediately. Simultaneously, ANF ( $\alpha$-hANF, Peninsula Laboratories, Inc., Belmont, CA) was infused intravenously at a rate of $12.5-25.0 \mathrm{ng} / \mathrm{kg}$ per min to achieve a plasma concentration similar to that observed during rapid ventricular pacing. In this group, only animals in which exogenous ANF mimicked plasma ANF in group I underwent further renal and endocrine analysis. After $15 \mathrm{~min}$ of stabilization, a 30-min clearance (EP) was obtained. At the end of $30 \mathrm{~min}$, the infusion of ANF was terminated and the vascular occluder was deflated. The clearance during RP was collected as above.

\section{Methods of measurement}

During each clearance, the following hemodynamic data were collected: MAP, right atrial pressure (RAP), pulmonary artery capillary wedge pressure (PCWP) (to assess left atrial pressure), heart rate (HR), renal blood flow (RBF), and cardiac output (CO). CO was measured by thermodilution using American Edwards Cardiac Output model 9510-A computer. Systemic vascular resistance was calculated from MAP - RAP/CO $\times 80$. For each clearance, $\mathrm{CO}$ was determined in triplicate and averaged.

During each clearance period, arterial blood was collected for hormone analysis, electrolyte determination, and hematocrit. Blood for hormone analysis was placed in EDTA tubes, immediately placed on ice, and centrifuged at $2,500 \mathrm{rpm}$ at $3^{\circ} \mathrm{C}$. Plasma was separated and stored at $-20^{\circ} \mathrm{C}$ until assay. ANF was extracted by use of Bond-Elut (Denver, $\mathrm{CO}$ ) with a recovery of $86 \%$. ANF was measured by radioimmunoassay to $\alpha$-H-ANF (11). Interassay coefficient of variation was $9 \%$; intraassay coefficient of variation was $6 \%$. Plasma renin activity (PRA) was determined by radioimmunoassay using the method of Haber et al. (15). Aldosterone (immuno-aldosterone-iodine-125 Kit, Pantex D18, Santa Monica, CA) levels were determined after dichloromethane extraction by radioimmunoassay ( $95 \%$ recovery). Arginine vasopressin was determined by radioimmunoassay as previously reported from our laboratory (5). Serum and urine sodium concentrations were determined during each clearance by use of ion-selective electrodes using a model E2A analyzer (Beckman Instruments, Inc., Brea, CA). Glomerular filtration rate was determined by the clearance of inulin. Plasma and urine inulin concentrations were measured by the anthrone method (16).

\section{Data analysis}

Since the variances of the average changes in hormonal and hemodynamic variables were not homogenous (Bartlett's test), a nonparametric procedure (Kruskal-Wallis test) was used to test for difference among the means. If the Kruskal-Wallis test detected a significant difference $(P<0.05)$, a nonparametric multiple-comparison test was used to compare the means.

\section{Results}

Table I reports cardiovascular, renal, and endocrine function in all three groups.

Group 1. A $15 \%$ reduction of MAP was achieved and maintained for $30 \mathrm{~min}$ by RVP; the pressure reduction achieved was $15 \pm 2 \%$ (Fig. 1). The $\mathrm{CO}$ was reduced by 33 $+3 \%$. Systemic vascular resistance did not significantly change. Both the right atrial and the pulmonary wedge pressure increased in response to rapid ventricular pacing. Plasma ANF increased from a control value of $58 \pm 7$ to $396+39$ $\mathrm{pg} / \mathrm{ml}$ in association with a decrease in vasopressin from $9.8 \pm 1.1$ to $4.8 \pm 1.3 \mathrm{pg} / \mathrm{ml}$. Despite reductions in arterial pressure as well as $\mathrm{CO}$, no reductions in urine output, sodium excretion, fractional sodium excretion, plasma renin activity, aldosterone, glomerular filtration rate, and RBF were observed.

Group 2. A $15 \%$ reduction of MAP was achieved and maintained for $30 \mathrm{~min}$ by constriction of the thoracic inferior vena cava; the arterial pressure reduction achieved was $14 \pm 1 \%$ (Fig. 1). The cardiac output was reduced by $42 \pm 5 \%$ and systemic vascular resistance significantly increased. The right atrial pressure decreased while the pulmonary wedge pressure did not change. Constriction of the thoracic vena cava did not change the plasma ANF level but vasopressin increased from $6.1 \pm 1.1$ to $10.1 \pm 1.6 \mathrm{pg} / \mathrm{ml}$. In spite of similar reductions in systemic arterial pressure and $\mathrm{CO}$ when compared with group 1 , there were marked reductions in urine output, sodium excretion, and fractional excretion of sodium. In contrast to rapid ventricular pacing, constriction of the thoracic vena cava caused significant increases in plasma renin activity and aldosterone. There was no change in glomerular filtration rate with, however, a decrease in RBF.

Group 3. A $15 \%$ reduction of MAP was intended and maintained for $30 \mathrm{~min}$ by simultaneous infusion of ANF and constriction of the thoracic vena cava; the arterial pressure reduction achieved was $15+1 \%$ (Fig. 1). The cardiac output was reduced by $41 \pm 3 \%$. Systemic vascular resistance did not change. The right atrial pressure decreased while the pulmo- 
Table I. Cardiovascular, Renal, and Endocrine Function

\begin{tabular}{|c|c|c|c|}
\hline & Baseline & Experimental & Recovery \\
\hline \multicolumn{4}{|c|}{ Right RVP (group I, $n=5$ ) } \\
\hline MAP $(m m ~ H g)$ & $119 \pm 3$ & $102 \pm 3^{*}$ & $116 \pm 4$ \\
\hline $\mathrm{CO}($ liter/min $)$ & $3.8 \pm 0.2$ & $2.5 \pm 0.2^{*}$ & $3.6 \pm 0.2$ \\
\hline $\operatorname{SVR}(R U)$ & $2468 \pm 481$ & $3011 \pm 521$ & $2522 \pm 461$ \\
\hline $\operatorname{RAP}(m m ~ H g)$ & $2.3 \pm 0.4$ & $5.3 \pm 0.6^{*}$ & $2.0 \pm 0.5$ \\
\hline PCWP $(m m H g)$ & $6.9 \pm 0.4$ & $18.7 \pm 1.8^{*}$ & $7.5 \pm 1.2$ \\
\hline GFR $(\mathrm{ml} / \mathrm{min})$ & $40 \pm 2$ & $42 \pm 1$ & $41 \pm 2$ \\
\hline $\mathrm{RBF}(\mathrm{ml} / \mathrm{min})$ & $208 \pm 20$ & $182 \pm 19$ & $201 \pm 21$ \\
\hline $\mathrm{U}_{\mathrm{Na}} \mathrm{V}(\mu e q / \mathrm{min})$ & $22 \pm 8$ & $35 \pm 11$ & $33 \pm 9$ \\
\hline $\mathrm{FE}_{\mathrm{Na}}(\%)$ & $0.4 \pm 0.2$ & $0.6 \pm 0.2$ & $0.7 \pm 0.2$ \\
\hline ANF $(p g / m l)$ & $58 \pm 7$ & $396 \pm 40^{*}$ & $63 \pm 9$ \\
\hline PRA $(n g / m l p e r h)$ & $2.1 \pm 0.5$ & $5.1 \pm 2.0$ & $3.5 \pm 2.0$ \\
\hline $\mathrm{PA}(n g / d l)$ & $11.9 \pm 1.1$ & $11.4 \pm 1.6$ & $12.5 \pm 0.7$ \\
\hline $\operatorname{AVP}(p g / m l)$ & $9.8 \pm 1.1$ & $4.8 \pm 1.3^{*}$ & $10.0 \pm 1.8$ \\
\hline \multicolumn{4}{|c|}{ TIVCC (group II, $n=5$ ) } \\
\hline MAP $(m m ~ H g)$ & $120 \pm 2$ & $102 \pm 2 *$ & $116 \pm 4$ \\
\hline $\mathrm{CO}($ liter/min $)$ & $3.3 \pm 0.2$ & $1.7 \pm 0.1^{*}$ & $3.4 \pm 0.3$ \\
\hline $\operatorname{SVR}(R U)$ & $2822 \pm 462$ & $4816 \pm 722^{*}$ & $2695 \pm 476$ \\
\hline $\operatorname{RAP}(m m H g)$ & $1.7 \pm 0.5$ & $-0.3 \pm 0.4^{*}$ & $1.6 \pm 0.4$ \\
\hline PCWP $(m m ~ H g)$ & $7.8 \pm 0.7$ & $6.2 \pm 0.8$ & $8.1 \pm 0.6$ \\
\hline GFR $(\mathrm{ml} / \mathrm{min})$ & $43 \pm 3$ & $41 \pm 3$ & $42 \pm 3$ \\
\hline $\mathrm{RBF}(\mathrm{ml} / \mathrm{min})$ & $204 \pm 18$ & $145 \pm 21^{*}$ & $200 \pm 24$ \\
\hline $\mathrm{U}_{\mathrm{Na}} \mathrm{V}(\mu e q / \min )$ & $34 \pm 18$ & $2.5 \pm 1.2^{*}$ & $34 \pm 10$ \\
\hline $\mathrm{FE}_{\mathrm{Na}}(\%)$ & $0.6 \pm 0.2$ & $0.04 \pm 0.02^{*}$ & $0.6 \pm 0.3$ \\
\hline $\mathrm{ANF}(p g / m l)$ & $62 \pm 7$ & $63 \pm 8$ & $67 \pm 9$ \\
\hline PRA $(n g / m l p e r h)$ & $2.5 \pm 1.4$ & $9.3 \pm 3.0^{*}$ & $2.6 \pm 1.1$ \\
\hline PA $(n g / d l)$ & $7.6 \pm 1.9$ & $24.4 \pm 4.4^{*}$ & $7.6 \pm 0.9$ \\
\hline $\operatorname{AVP}(p g / m l)$ & $6.1 \pm 1.1$ & $10.1 \pm 1.6^{*}$ & $5.8 \pm 1.4$ \\
\hline \multicolumn{4}{|c|}{ TIVCC (group III, $n=5$ ) } \\
\hline MAP $(m m H g)$ & $114 \pm 9$ & $90 \pm 8^{*}$ & $120 \pm 7$ \\
\hline $\mathrm{CO}($ liter/min $)$ & $3.7 \pm 0.3$ & $2.4 \pm 0.2^{*}$ & $3.2 \pm 0.2$ \\
\hline $\operatorname{SVR}(R U)$ & $2425 \pm 411$ & $2885 \pm 476$ & $2871 \pm 433$ \\
\hline $\operatorname{RAP}(m m H g)$ & $2.0 \pm 0.3$ & $0.5 \pm 0.4^{*}$ & $1.8 \pm 0.4$ \\
\hline PCWP $(m m H g)$ & $7.4 \pm 0.4$ & $6.4 \pm 0.5$ & $7.4 \pm 0.6$ \\
\hline GFR $(\mathrm{ml} / \mathrm{min})$ & $43 \pm 4$ & $41 \pm 3$ & $41 \pm 3$ \\
\hline $\mathrm{RBF}(\mathrm{ml} / \mathrm{min})$ & $239 \pm 24$ & $214 \pm 26$ & $224 \pm 26$ \\
\hline $\mathrm{U}_{\mathrm{Na}} \mathrm{V}(\mu e q / \mathrm{min})$ & $18 \pm 5$ & $35 \pm 14$ & $45 \pm 22$ \\
\hline $\mathrm{FE}_{\mathrm{Na}}(\%)$ & $0.3 \pm 0.1$ & $0.6 \pm 0.3^{*}$ & $0.5 \pm 0.4$ \\
\hline $\mathrm{ANF}(p g / m l)$ & $62 \pm 12$ & $408 \pm 43^{*}$ & $84 \pm 12$ \\
\hline PRA $(n g A I / m l / h)$ & $3.0 \pm 1.2$ & $5.2 \pm 2.0$ & $2.9 \pm 1.4$ \\
\hline $\mathrm{PA}(n g / d l)$ & $14.3 \pm 3.5$ & $14.7 \pm 5.2$ & $16.0 \pm 5.8$ \\
\hline $\operatorname{AVP}(p g / m l)$ & $9.4 \pm 0.6$ & $10.9 \pm 1.0$ & $6.5 \pm 1.2$ \\
\hline
\end{tabular}

Values are mean \pm SE. Abbreviations: MAP, mean arterial pressure; RBF, renal blood flow; GFR, glomerular filtration rate; CO, cardiac output; RAP, right atrial pressure; PCWP, pulmonary capillary wedge pressure; $S V R$, systemic vascular resistance; $U_{\mathrm{Na}} \mathrm{V}$, urinary sodium excretion; $\mathrm{FE}_{\mathrm{Na}}$, fractional excretion of sodium; $\mathrm{ANF}$, atrial natriuretic factor; PRA, plasma renin activity; PA, plasma aldosterone; AVP, arginine vasopressin. $P$ values compare results with control: ${ }^{*} P<0.05$

nary wedge pressure did not change. Exogenous infusion of ANF was designed to mimic endogenous ANF level $(396+39$ $\mathrm{pg} / \mathrm{ml}$ ) during RVP; the ANF level achieved was $407 \pm 43$ $\mathrm{pg} / \mathrm{ml}$. Vasopressin remained unchanged. The decreases in urine output, sodium excretion, RBF, and fractional excretion
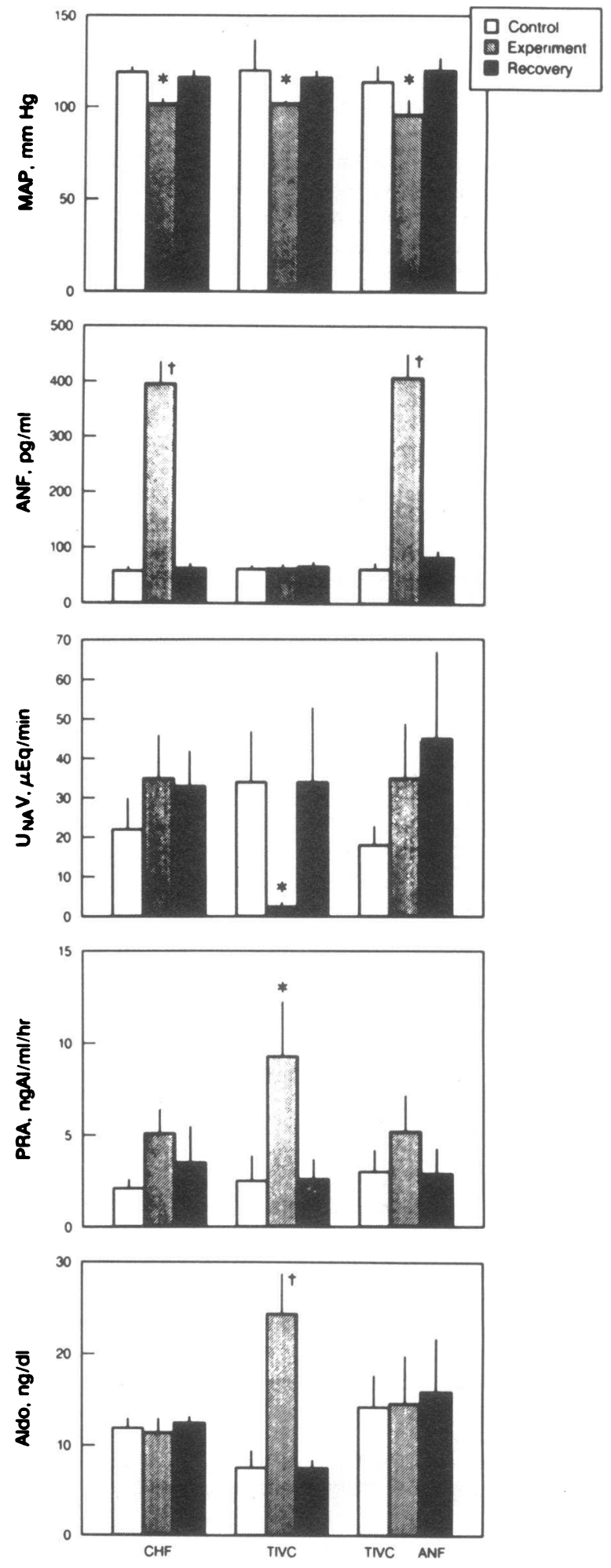

p. 0.05 Experimental or recovery vs. control tp. 0.01 Experimentel or recovery ve. control

Figure 1. Effect of rapid right ventricular pacing (CHF), thoracic inferior vena caval constriction (TIVCC), and TIVCC plus exogenous ANF on mean arterial pressure (MAP), plasma ANF, urinary sodium excretion $\left(\mathrm{U}_{\mathrm{NA}} \mathrm{V}\right)$, plasma renin activity (PRA), and plasma aldosterone. All data are expressed as mean $\pm \mathrm{SE}$.

of sodium, as well as the increases of PRA and aldosterone in response to constriction of thoracic vena cava, was prevented when ANF was infused simultaneously. 


\section{Discussion}

The present study was undertaken to investigate the functional role of ANF in acute CHF. RVP decreased CO and systemic arterial pressure and increased cardiac filling pressures. Thus, in this model of acute CHF, reductions in systemic arterial pressure, and hence renal perfusion pressure, should result in retention of sodium and water with activation of the RAAS. However, despite a $15 \%$ reduction in systemic arterial pressure and $33 \%$ reduction in $\mathrm{CO}$, no decreases in urine output and sodium excretion during right RVP were observed. Moreover, renin and aldosterone were not significantly increased. In contrast, marked decreases in urine output and sodium excretion in response to similar reductions in systemic arterial pressure and cardiac output during constriction of the thoracic vena cava was demonstrated in association with activation of both renin and aldosterone.

This paradox in cardiorenal and endocrine function in these two models of acute low output failure can be explained by the simultaneous increases in ANF and/or atrial pressures during RVP which did not occur during constriction of thoracic inferior vena cava. Moreover, increased atrial pressures not only release ANF but also stimulate low-pressure cardiopulmonary receptors with inhibition of vasopressin release as was observed in the present study (17). Both increased plasma ANF and stimulation of cardiopulmonary receptors have been reported to cause natriuresis and diuresis and inhibition of renin release (17-19). Thus, the differential changes of renal and endocrine function during rapid ventricular pacing and constriction of the thoracic vena cava could be due to either ANF or cardiopulmonary receptor activation.

The use of exogenous ANF infused during constriction of thoracic vena cava to mimic the endogenous ANF level of RVP elucidates, however, the relative role of ANF versus cardiopulmonary receptors in the preservation of sodium and water excretion and modulation of the RAAS in response to systemic hypotension during rapid ventricular pacing. Our results indicate that ANF prevented the reductions in urine output and sodium excretion in response to systemic hypotension. This is supported by group 3 in which in the absence of atrial stretch during TIVCC but during similar reductions in arterial pressure and $\mathrm{CO}$ was associated with a maintenance of sodium excretion. The mechanism of the preservation of sodium excretion in acute CHF most likely represents a tubular action of the peptide at or beyond the proximal tubule inasmuch as glomerular filtration rate was not different between the pacing and caval constriction models. Thus, ANF may play a major role in the preservation of the electrolyte excretion during systemic hypotension caused by rapid ventricular function, a model of acute congestive heart failure.

The RAAS contributes importantly to the regulation of fluid and electrolyte balance and in the pathogenesis of congestive heart failure $(20,21)$. Systemic hypotension is a potent stimulus for activation of this vasoconstrictive-sodium retaining system. Indeed, our results demonstrated that renin and aldosterone increased significantly in response to arterial hypotension caused by constriction of the thoracic vena cava which is consistent with previous reports (20-22). The greater activation observed during TIVCC may have, in part, occurred in association with a greater decrease in $\mathrm{CO}$ and $\mathrm{RBF}$. However, the increases in renin and aldosterone were prevented when the systemic hypotension was associated with a simultaneous increase of either endogenous ANF or exoge- nous ANF. Thus, ANF possibly also plays an important role in the regulation of the RAAS during acute reductions in $\mathrm{CO}$ and arterial pressure. Further, these studies may support the speculation of Hodgman et al., who suggest that the mechanism of lack of activation of the RAAS observed in acute myocardial infarction in the rat is secondary to activation of ANF (6).

The response in vasopressin also provides insight into the relative contribution of atrial stretch and/or ANF in vasopressin release as well as the role of vasopressin in the renal response to acute CHF. The decrease in vasopressin during acute $\mathrm{CHF}$ with an associated increase in atrial pressure and ANF suggest a synergistic role for cardiopulmonary reflexes and ANF as inhibitors to vasopressin release. The marked increase in vasopressin in TIVCC and hypotension with a decrease in right atrial pressure and no change in ANF supports this interpretation. The lack of suppression of vasopressin with TIVCC and exogenous ANF suggests that atrial stretch may be more important than ANF in vasopressin control. That the response in sodium excretion, however, were similar during acute pacing induced CHF and TIVCC with exogenous ANF but with different responses in vasopressin, suggests the renal response is not modulated by vasopressin.

Preliminary studies in the dog support a role for ANF in the preservation of RBF (14) in acute CHF. In the present study, RBF decreased significantly in response to systemic hypotension caused by constriction of the thoracic vena cava. However, the reduction was prevented when the systemic hypotension was associated with a simultaneously increased endogenous or exogenous ANF level. Again, this supports a functional role for ANF in the regulation of the renal circulation in response to reduction in arterial blood pressure and $\mathrm{CO}$ during acute $\mathrm{CHF}$.

The present studies are consistent with preliminary observations suggesting a functional role for elevated endogenous ANF in chronic CHF. Drexler et al. (23) have demonstrated an increase in peripheral vascular resistance in rats with chronic CHF produced by myocardial infarction when ANF antibodies were infused. This would suggest that ANF is an endogenous vasodilator in chronic CHF. In our present studies, a greater increase in systemic vascular resistance was observed in the TIVCC group in which ANF was not increased. Taken together, ANF may serve to offset increases in systemic vascular resistance in CHF during reductions in CO. From the perspective of sodium excretion, Awazu et al. (24) also reported preliminary studies in the same rat model of chronic congestive heart failure that administration of ANF antibodies resulted in sodium and water retention without changes in glomerular filtration rate consistent with a homeostatic role.

The present studies confirm and extend these previous preliminary studies. Most importantly, by mimicking endogenous levels of elevated ANF by infusion of exogenous ANF in the thoracic inferior vena caval dogs, we can differentiate between the contribution of atrial stretch and activation of neurogenic reflexes as compared to ANF in mediating the maintenance of sodium excretion and possible suppression of activation of the RAAS in acute CHF. Indeed, these studies are consistent with the recent studies of Greenwald and colleagues (25), who have demonstrated a role for ANF as an acute regulator of intravascular volume during states of acute volume overload. Caution should be exercised regarding the complexity of various opposing vasoactive and sodium regulating systems operative in $\mathrm{CHF}$; yet, the studies support a contributing 
homeostatic role for endogenous ANF in acute CHF. The relevance of the present studies to chronic congestive heart failure must be interpreted with caution as receptor down-regulation may contribute to a blunted target organ responsiveness during chronic elevation of atrial peptide.

In summary, these studies demonstrate that endogenous ANF serves as an important physiologic volume regulator in acute CHF. Its role may be to prevent acute intravascular volume overload by maintaining sodium and water excretion and possibly suppress activation of the RAAS despite the potent stimulus of arterial hypotension.

\section{Acknowledgments}

This research was supported by grants HL-36634 and HL-07111 from the National Institutes of Health, 86-767, from the American Heart Association, and from the Rappaport and Mayo Foundations. Dr. Burnett is an Established Investigator of the American Heart Association.

\section{References}

1. Dietz, J. R. 1984. Release of natriuretic factor from rat heartlung preparations by atrial distention. Am. J. Physiol. 247:R1093R1096.

2. Edwards, B. S., R. S. Zimmerman, T. R. Schwab, D. M. Heublein, and J. C. Burnett, Jr. 1988. Atrial stretch, not pressure, is the principal determinant controlling the acute release of atrial natriuretic factor. Circ. Res. 62:191-195.

3. Burnett, J. C., Jr., J. P. Granger, and T. J. Opgenorth. 1984. Effects of synthetic atrial natriuretic factor on renal function and renin release. Am. J. Physiol. 247:F863-F869.

4. Maack, T., D. H. Marion, M. J. F. Camargo, H. D. Kleinert, J. H. Laragh, E. D. Vaughan, and S. A. Atlas. 1984. Effects of synthetic atrial natriuretic factor on kidney function and the renin-aldosterone system in the dog. Am. J. Med. 77:1069-1075.

5. Zimmerman, R., J. Schirger, B. Edwards, T. Schwab, D. M. Heublein, and J. C. Burnett, Jr. 1987. Cardio-renal-endocrine dynamics during step-wise infusion of physiologic and pharmacologic concentrations of atrial natriuretic factor in the dog. Circ. Res. 60:6369.

6. Hodman, G. P., M. Kohzuki, L. G. Howes, E. Sumithran, K. Tsunoda, and C. I. Johnston. 1988. Neurohumoral responses to chronic myocardial infarction in rats. Circulation. 78:376-381.

7. Michel, J., A. Lattion, J. Salzman, M. Cerol, M. Philippe, J. Camilleri, and P. Corvol. 1988. Hormonal and cardiac effects of converting enzyme inhibition in rat myocardial infarction. Circ. Res. 62:641-650.

8. Ding, J., G. Thibault, J. Gutkowska, R. Garcia, T. Karabatsos, G. Jasmin, J. Genest, and M. Cantin. 1987. Cardiac and plasma atrial natriuretic factor in experimental congestive heart failure. Endocrinology. 121:248-257.

9. Edwards, B. S., D. M. Ackermann, M. E. Lee, G. S. Reeder, L. E. Wold, and J. C. Burnett, Jr. 1988. Identification of atrial natriuretic factor within ventricular tissue in hamsters and humans with congestive heart failure. J. Clin. Invest. 81:82-86.

10. Cody, R. J., S. A. Atlas, J. H. Laragh, S. H. Kubo, A. B. Covit,
K. S. Ryman, A. Shaknovich, K. Pondolfino, M. Clark, M. J. F. Camargo, R. M. Scarborough, and J. A. Lewicki. 1986. Atrial natriuretic factor in normal subjects and heart failure patients: plasma levels and renal, hormonal, and hemodynamic responses to peptide infusion. $J$. Clin. Invest. 78:1362-1374.

11. Burnett, J. C., Jr., P. C. Kao, D. C. Hu, D. W. Heser, D. Heublein, J. P. Granger, T. J. Opgenorth, and G. S. Reeder. 1986. Atrial natriuretic peptide elevation in congestive heart failure in the human. Science (Wash. DC). 231:1145-1147.

12. Shenker, Y, R. S. Sider, E. A. Ostafin, and R. J. Grekin. 1985. Plasma levels of immunoreactive atrial natriuretic factor in healthy subjects and in patients with edema. J. Clin. Invest. 76:1684-1687.

13. Itoh, H., K. Nakao, M. Mukoyama, A. Sugawara, Y. Saito, N. Morii, T. Yamada, S. Shiono, H. Arai, and H. Imura. 1988. Secretion of N-terminal fragment of gamma-human atrial natriuretic polypeptide. Hypertension. 11:152-I56.

14. Miller, W. L., B. S. Edwards, R. S. Zimmerman, and J. C. Burnett, Jr. 1987. Attenuated natriuresis to atrial natriuretic factor during tachycardia induced reduction in renal perfusion pressure. Proceedings of the Congress on Biological Action of Atrial Peptides. Raven Press, New York. 192A. (Abstr.)

15. Haber, E., O. Loerner, T. Page, B. Kleman, and A. Purnode. 1969. Application of a radioimmunoassay for angiotensin I to the physiologic measurement of plasma renin activity in normal human subjects. J. Clin. Endocrinol. 29:1329-1355.

16. Fuhr, J., J. Kachzmarczyk, and C. D. Kruttgen. 1955. Eine einfache colorimetrische Method zur Insulin bestimmung Nierenclearance untersuchungen bei Stoffwech-selgessunden und Diabetikern. Klin. Wochenschr. 33:729-730.

17. Metzler, C. H., M. E. Lee, T. N. Thrasher, and D. J. Ramsay. 1986. Increased right or left atrial pressure stimulates release of atrial natriuretic peptides in conscious dogs. Endocrinology. 119:2396-2398.

18. Thoren, P., A. Mark, D. Morgan, P. O'Neill, P. Needleman, and M. Brody. 1986. Activation of vagal depressor reflexes by atriopeptins inhibits renal sympathetic nerve activity. Am. J. Physiol. 251:H1252-H1259.

19. Gauer, O. H., J. P. Henry, and C. Behn. 1970. The regulation of extracellular fluid volume. Annu. Rev. Physiol. 32:547-595.

20. Watkins, L., J. A. Burton, E. Haber, J. R. Cant, F. W. Smith, and A. C. Barger. 1976. The renin-angiotensin-aldosterone system in congestive failure in conscious dogs. J. Clin. Invest. 57:1606-1614.

21. Davis, J. O., M. M. Pechet, W. S. Ball, Jr., and M. J. Goodkin. 1957. Increased aldosterone secretion in dogs with right-sided congestive heart failure and in dogs with thoracic inferior vena cava constriction. J. Clin. Invest. 36:689-697.

22. Scriven, T. A., and J. C. Burnett, Jr. 1985. Effects of synthetic atrial natriuretic peptide on renal function and renin release in acute experimental heart failure. Circulation. 72:892-897.

23. Drexler, H., C. Hirth, F. Morich, C. Traub, and G. Maio. 1987. Vasodilatory action of endogenous ANP in chronic congestive heart failure as determined by monoclonal ANP-antibodies. Circulation. 76:II 532A.

24. Awazu, M., H. Imada, V. Kon, T. Inagami, and I. Ichikawa. 1988. Assessment of the functional role of endogenous atrial natriuretic peptide by purified anti-ANP antibody: study in rat model of congestive heart failure. Kidney Int. 33:253.

25. Greenwald, J. E., M. Kakata, M. L. Michener, S. D. Sides, and P. Needleman. 1988. Is atriopeptin a physiological or pathophysiological substance? J. Clin. Invest. 81:1036-1041. 